\title{
Blow up Property of the Solution for Quasi Linear Parabolic Equations and Its Application
}

\author{
Zhongwei Zha \\ Department of Mathematics, Chongqing Three Gorges University \\ Chongqing 404000, China \\ E-mail: sxxyzzw@163.com
}

This work was partially supported by NSF of China, KJF(1011xx) of CQEC

\begin{abstract}
This dissertation is to discuss the initial-boundary value problem under the third nonlinear boundary condition for a kind of quasi-linear parabolic equations. To apply the maximum value theory and convex method, it is proved that the blowing up of solution in the definite time. And for application, this paper research into a mathematics model of fluids in porous medium. And have got the blowing up behavior for the problem in limited time.
\end{abstract}

Keywords: Quasi linear parabolic equation, Initial-boundary balue problem, Blowing up of solution, Flow dynamics

AMS Mathematics Subject Classification (2000): 35K57

\section{Introduction}

It is significant practically for the research that the solution of mathematics physical partial differential coefficient equation (Developmental Equation) which is related to the time variable $t$ and appear in engineering dynamics such as the limited swing wave spreading, mixed gas burning, fluent mechanics and reaction diffusing, blows up in limited time. Because, whether the research of unitary characters (e g. stability) of fixed solution problem for nonlinear developmental equation or finding the methods of numerical value computing, both base on unitary existence of the solution. If the obtained solution blows up in limited time (the solution is to infinite in limited time), however, such blowing up behavior is not permit by the relevant engineer dynamics model, this shows the engineer dynamics model is questionable and it should be modified. If such blowing up behavior permit the studied engineer dynamics problem, we must compute in a more extensive function since the relevant engineering processes will keep on developing by no means of ending at a certain moment.

This dissertation discuss the original boundary value problem for the quasi-lineal parabolic equation with the third type nonlinear boundary condition:

$$
\left\{\begin{array}{lc}
\frac{\partial u}{\partial t}=\sum_{i, j=1}^{n} \frac{\partial}{\partial x_{i}}\left(a_{i j}(x) \frac{\partial u}{\partial x_{j}}\right)+\sum_{i, j=1}^{n}\left(a_{i j}(x) \frac{\partial u}{\partial x_{i}} \frac{\partial u}{\partial x_{j}}+b(x) u+f\left(x, t, u, \nabla_{x} u\right),\right. & D \times(0, T) \\
\beta(x) \frac{\partial u}{\partial v}+u=g\left(x, t, u, \nabla_{x} u\right) & \partial D \times(0, T) \\
u(x, 0)=u_{0}(x) & \bar{D}
\end{array}\right.
$$

$\mathrm{D}$ is the limited domain within $R^{n}, x=\left(x_{1}, x_{2}, \cdots, x_{n}\right), \beta(x)>0, a_{i j}(x)=a_{j i}(x)$ is the continual differentiable on $\bar{D}$, to $\zeta=\left(\zeta_{1}, \zeta_{2}, \cdots, \zeta_{n}\right) \in R^{n}$, it exists constant $\alpha>0$, cause

$$
\sum_{i, j=1}^{n} a_{i j} \zeta_{i} \zeta_{j} \geq \alpha \sum_{i=1}^{n} \zeta_{i}^{2}
$$

$\frac{\partial u}{\partial v}=\sum_{i, j=1}^{n} a_{i j} \frac{\partial u}{\partial x_{j}} n_{i}$ is the normal differential coefficient related to matrix $\left(a_{i j}(x)\right)$ on suitable slick boundary $\partial D$. $n=$ $\left(n_{1}, n_{2}, \cdots, n_{n}\right)$ is the unit outer normal of $\partial D, \nabla_{x} u=\left(\frac{\partial u}{\partial x_{1}}, \frac{\partial u}{\partial x_{2}}, \cdots, \frac{\partial u}{\partial x_{n}}\right)$. It can also simplify to $f_{\nabla_{x} u}^{\prime}=\left(\frac{\partial f}{\partial q_{1}}, \frac{\partial f}{\partial q_{2}}, \cdots, \frac{\partial f}{\partial q_{n}}\right)$. $q_{1}=\frac{\partial u}{\partial x_{1}}, q_{2}=\frac{\partial u}{\partial x_{2}}, \cdots, q_{n}=\frac{\partial u}{\partial x_{n}}, \frac{\partial\left(\nabla_{x} u\right)}{\partial t}=\left(\frac{\partial q_{1}}{\partial t}, \frac{\partial q_{2}}{\partial t}, \cdots, \frac{\partial q_{n}}{\partial t}\right)$.

Dissertation (Pao C.V., 1980) is to discuss the blowing up character for the solution of problem (1) while $f=\lambda\left(e^{a u}-b\right)$ (where $\lambda, a, b$ are non-negative constant) and $g=0$. Suppose $b(x) \equiv 0$ and $f(u)$ is just the function of $u$, literatures (Deng Jucheng, 1987; Chipot M., 1989) studies when $g=0$ and $g=g(u)$, solution of problem (1) blow up in limited time; on the bases of $b(x) \neq 0$ and $f$ is a function of $x, t$, and $u$, literature (Zheng Zongmu, 1987) discusses blow up problem of boundary condition $g \equiv 0$. The problem discussed in literatures (Gomez J.L., 1991; Migoguchi N., 1997; Zhang Hailiang, 2002; Li Junfeng, 2002) is special situation of (1). This dissertation extends the problem studied in literature (Zha Zhongwei, 1992), accordingly original boundary value problem has more comprehensive physical backgrounds.

Suppose: 
$\left.A_{1}\right) u_{0}(x) \in C^{2}(\bar{D}), u_{0}(x) \geq 0$.

$\left.A_{2}\right) a_{i j}(x), b(x)>0$ is a suitable slick function, function $f(x, t, p, q) \in C^{1}\left[R^{n} \times[0, T) \times R \times R\right], f, f_{t}^{\prime}>0$, quantitative product $f_{q}^{\prime} \cdot q_{t}^{\prime}=\frac{\partial f}{\partial q_{1}} \cdot \frac{\partial q_{1}}{\partial t}+\frac{\partial f}{\partial q_{2}} \cdot \frac{\partial q_{2}}{\partial t}+\cdots+\frac{\partial f}{\partial q_{n}} \cdot \frac{\partial q_{n}}{\partial t}$ is non-negative, and exists constant $h>1$, which lead to $f_{p}^{\prime}-(h-1) f \geq 0$ is available to any $p \geq 0$.

$\left.A_{3}\right) g(x, t, p, q) \in C^{1}\left[R^{n} \times[0, T) \times R \times R\right], g_{t}^{\prime} \geq 0$, quantitative product $g_{q}^{\prime} \cdot q_{t}^{\prime}$ is non-negative; when $p \geq 0, g \leq 0$; when $p<0, g>0$. to the same $h$, when $p \geq 0$, it has the inequality $g_{p}^{\prime}<1$ and $g_{p}^{\prime}+(h-1) p-1 \geq 0$.

$\left.A_{4}\right)$ when $x \in \bar{D}, \sum_{i, j=1}^{n} \frac{\partial}{\partial x_{i}}\left(a_{i j} \frac{\partial e^{u_{0}}}{\partial x_{j}}+e^{u_{0}}\left[b u_{0}+f\left(x, 0, u_{0}, \nabla_{x} u_{0}\right)\right] \geq 0\right.$.

\section{The non-negative character of solution}

In order to prove the solution of problem (1) blowing up in limited time, we should apply some lemmas as follows.

Lemma 1 If A1) - A 3) satisfied on $\bar{D} \times[0, T)$, (1)'s solution $u(x, t)$ is non-negative.

Proof Take constant $K>\max \{b(x), x \in \bar{D}\}$, suppose $u(x, t)=V(x, t) e^{K t}$, then (1) become (3)

$$
\left\{\begin{array}{lc}
\frac{\partial V}{\partial t}=\sum_{i, j=1}^{n} \frac{\partial}{\partial x_{i}}\left(a_{i j} \frac{\partial V}{\partial x_{j}}\right)+\sum_{i, j=1}^{n} a_{i j} \frac{\partial V}{\partial x_{i}} \frac{\partial V}{\partial x_{j}} e^{K t}-(K-b) V+f\left(x, t, V e^{K t}, \nabla_{x} V e^{K t}\right) e^{-K t}, & D \times(0, T) \\
\beta(x) \frac{\partial V}{\partial v}+V=g\left(x, t, V e^{K t}, \nabla_{x} V e^{K t}\right) e^{-K t}, & D D \times(0, T) \\
V(x, 0)=u_{0}(x) . & \bar{D}
\end{array}\right.
$$

Obviously, we just need to prove solution of problem (3) $V(x, t)$ is non-negative on $\bar{D} \times[0, T)$. To apply counter evidence, providing $V(x, t)$ can take negative, then it must have a point $M_{0}\left(x_{0}, t_{0}\right)$ result in $V\left(M_{0}\right)$ the negative minimum value , according to (3) $V(x, 0)=u_{0}(x)$ to know $t_{0} \neq 0$.

If $M_{0} \in D \times(0, T)$, then $\left.\frac{\partial V}{\partial t}\right|_{M_{0}} \leq 0$. But since $\left.\frac{\partial V}{\partial x_{i}}\right|_{M_{0}} \leq 0,\left.\sum_{i, j=1}^{n} \frac{\partial}{\partial x_{i}}\left(a_{i j} \frac{\partial V}{\partial x_{j}}\right)\right|_{M_{0}} \geq 0$ and condition A2), we can know the right of (3)' first equation should be positive, which is illogical.

If $M_{0} \in \partial D \times(0, T)$, according to the maximum value principle we know $\left.\frac{\partial V}{\partial \gamma}\right|_{M_{0}} \leq 0$, the left of second equation in (3) is negative here, which is illogical with condition A3). So, it must have $u(x, t) \geq 0$ on $\bar{D} \times[0, T)$.

In order to discuss easily, we make function transform with $W=e^{u(x, t)}$, then problem (1) became (3):

$$
\left\{\begin{array}{lc}
\frac{\partial W}{\partial t}=\sum_{i, j=1}^{n} \frac{\partial}{\partial x_{i}}\left(a_{i j} \frac{\partial W}{\partial x_{j}}\right)+b W \ln W+W f\left(x, t, \ln W, \nabla_{x} \ln W\right), & D \times(0, T) \\
\beta(x) \frac{\partial W}{\partial v}+W \ln W=W g\left(x, t, \ln W, \nabla_{x} \ln W\right), & \partial D \times(0, T) \\
W(x, 0)=e^{u_{0}(x)} . & \bar{D}
\end{array}\right.
$$

Through Lemma 1 to know $W(x, t) \geq 1$.

Lemma 2 If condition A1)-A4) is satisfied, $W(x, t)$ is the solution of problem(2.2), and $S u p\{W(x, t),(x, t) \in \bar{D} \times[0, T)\}=$ $N<+\infty\}$, then it must has $\frac{\partial W}{\partial t} \geq 0$ on $\bar{D} \times[0, T)$.

Proof Let $Z(x, t)=\frac{\partial W}{\partial t}$, then $Z(x, t)$ satisfies original boundary value problem as follows:

$$
\left\{\begin{array}{lc}
\frac{\partial Z}{\partial t}=\sum_{i, j=1}^{n} \frac{\partial}{\partial x_{i}}\left(a_{i j} \frac{\partial Z}{\partial x_{j}}\right)+\left[b(\ln W+1)+f+f_{\ln W}^{\prime}\right] Z+W f_{t}^{\prime}+W f_{\nabla_{x} \ln W}^{\prime} \cdot \frac{\partial\left(\nabla_{x} \ln W\right)}{\partial t}, & D \times(0, T) \\
\beta(x) \frac{\partial Z}{\partial v}+\left(\ln W+1-g-g_{\ln W}^{\prime}\right) Z=W g_{t}^{\prime}+W g_{\nabla_{x} \ln W}^{\prime} \cdot \frac{\partial\left(\nabla_{x} \ln W\right)}{\partial t}, & \partial D \times(0, T) \\
Z(x, 0)=\sum_{i, j=1}^{n} \frac{\partial}{\partial x_{i}}\left(a_{i j} \frac{\partial e^{u_{0}}}{\partial x_{j}}\right)+\left[b u_{0}+f\left(x, 0, u_{0}, \nabla_{x} u_{0}\right)\right] e^{u_{0}} . & \bar{D}
\end{array}\right.
$$

Suppose $\operatorname{Sup}\left\{b p+f+f_{p}^{\prime}, 0 \leq p \leq N,(x, t) \in \bar{D} \times[0, T)\right\}=M<+\infty$, take $K>2 \max \{\max \{b(x), x \in \bar{D}, M\}$, then transform into $Z=V(x, t) e^{K t}$, then we get the original boundary value problem satisfied by $V(x, t)$ :

$$
\left\{\begin{array}{lc}
\frac{\partial V}{\partial t}=\sum_{i, j=1}^{n} \frac{\partial}{\partial x_{i}}\left(a_{i j} \frac{\partial V}{\partial x_{j}}\right)-\left[K-b-\left(b \ln W+f+f_{\ln W}^{\prime}\right)\right] V+W\left[f_{t}^{\prime}+f_{\nabla_{x} \ln W}^{\prime} \cdot \frac{\partial\left(\nabla_{x} \ln W\right)}{\partial t}\right] e^{-K t}, & D \times(0, T) \\
\beta(x) \frac{\partial V}{\partial v}+\left(\ln W+1-g-g_{\ln W}^{\prime}\right) V=W\left[g_{t}^{\prime}+g_{\nabla_{x} \ln W}^{\prime} \cdot \frac{\partial\left(\nabla_{x} \ln W\right)}{\partial t}\right] e^{-K t}, & \partial D \times(0, T) \\
V(x, 0)=\sum_{i, j=1}^{n} \frac{\partial}{\partial x_{i}}\left(a_{i j} \frac{\partial e^{u_{0}}}{\partial x_{j}}\right)+\left[b u_{0}+f\left(x, 0, u_{0}, \nabla_{x} u_{0}\right)\right] e^{u_{0}} . & \bar{D}
\end{array}\right.
$$

Similar with Lemma1's proving, it is easy to know the solution of problem (6), $V(x, t) \geq 0((x, t) \in \bar{D} \times[0, T))$, then $\frac{\partial W}{\partial t}=V(x, t) e^{k t} \geq 0$.

Moreover, one of conclusions in literature (Guan Zhicheng, 1984) will be applied, that is

Lemma 3 Suppose $J(t) \in C^{2}\left[t_{0},+\infty\right), J\left(t_{0}\right)>0, J^{\prime}\left(t_{0}\right)<0, J^{\prime \prime}(t) \leq 0\left(t_{0} \leq t+\infty\right)$, then exists $T_{0}\left(t_{0}<T_{0}<t_{0}-\frac{J\left(t_{0}\right)}{J^{\prime}\left(t_{0}\right)}\right)$,result in $J\left(T_{0}\right)=0$. 


\section{Blowing up Property of solution}

With the available condition of A1) - A4), if unitary slick solution of original boundary value problem $(1), u(x, t) \in$ $C^{3,2}[D \times(0, T)] \cap C^{2,1}[\bar{D} \times[0, T)]$, then $T$ can not excess a certain value, that is

Theorem Suppose condition A1)-A4) is satisfied, $u(x, t) \in C^{3,2}[D \times(0, T)] \cap C^{2,1}[\bar{D} \times[0, T)]$ is a solution of (1), then exists $T_{0}\left(0<T_{0} \leq T\right)$, result in

$$
\lim _{t \rightarrow T_{0}^{-}} \operatorname{Sup}\{u(x, t), x \in \bar{D}\}=+\infty
$$

Proof We just need to prove existing limited moment $T_{0}$, result in the solution of question (4) $W(x, t)$ blow up at $T_{0}$. That is

$$
\Phi(t)=\int_{D} \frac{1}{h+1} W^{h+1} d x
$$

There into, $h>1$ is the constant in condition A2). Finding differential coefficient with both sides of equation (7), we obtain

$$
\Phi^{\prime}(t)=\int_{D} W^{h} \frac{\partial W}{\partial t} d x
$$

Through lemma 1, lemma 2 to know

$$
\begin{gathered}
\Phi(0)=\left.\int_{D} \frac{1}{h+1} W^{h+1}\right|_{t=0} d x>0 \\
\Phi^{\prime}(0)=\left.\int_{D} W^{h} \frac{\partial W}{\partial t}\right|_{t=0} d x>0
\end{gathered}
$$

To take first equation of (4) into (8), then

$$
\Phi^{\prime}(t)=\int_{D} W^{h} \sum_{i, j=1}^{n} \frac{\partial}{\partial x_{i}}\left(a_{i j} \frac{\partial W}{\partial x_{j}}\right) d x+\int_{D} W^{h+1}\left[b \ln W+f\left(x, t, \ln W, \nabla_{x} \ln W\right)\right] d x
$$

Applying subsection integral to the first integral of the right side of above equation, we obtain:

$$
\begin{gathered}
\Phi^{\prime}(t)=\int_{\partial D} W^{h} \sum_{i, j=1}^{n}\left(a_{i j} \frac{\partial W}{\partial x_{j}}\right) n_{i} d s \\
-h \int_{D} W^{h-1} \sum_{i, j=1}^{n} a_{i j} \frac{\partial W}{\partial x_{j}} \frac{\partial W}{\partial x_{i}} d x+\int_{D} W^{h+1}\left[b \ln W+f\left(x, t, \ln W, \nabla_{x} \ln W\right)\right] d x
\end{gathered}
$$

there into $d s$ is the area element of $\partial D$. To find differential coefficient with equation (11) concerning $t$, we obtain

$$
\begin{gathered}
\Phi^{\prime \prime}(t)=h \int_{\partial D} W^{h-1} \frac{\partial W}{\partial t} \sum_{i, j=1}^{n} a_{i j} \frac{\partial W}{\partial x_{j}} n_{i} d s+\int_{\partial D} W^{h} \sum_{i, j=1}^{n}\left(a_{i j} \frac{\partial^{2} W}{\partial x_{j} \partial t}\right) n_{i} d s \\
-h(h-1) \int_{D} W^{h-2} \frac{\partial W}{\partial t} \sum_{i, j=1}^{n} a_{i j} \frac{\partial W}{\partial x_{j}} \frac{\partial W}{\partial x_{i}} d x-2 h \int_{D} W^{h-1} \sum_{i, j=1}^{n} \frac{\partial^{2} W}{\partial x_{j} \partial t} \frac{\partial W}{\partial x_{i}} d x \\
+(h+1) \int_{D} b W^{h} \frac{\partial W}{\partial t} \ln W d x+(h+1) \int_{D} W^{h} \frac{\partial W}{\partial t} f d x+\int_{D} b W^{h} \frac{\partial W}{\partial t} d x \\
+\int_{D} W^{h+1} f_{t}^{\prime} d x+\int_{D} W^{h} \frac{\partial W}{\partial t} f_{\ln W}^{\prime} d x+\int_{D} W^{h+1} f_{\nabla_{x} \ln W}^{\prime} \cdot \frac{\partial\left(\nabla_{x} \ln W\right)}{\partial t} d x
\end{gathered}
$$

In addition, if to find differential coefficient with both sides of (8) directly, we obtain

$$
\Phi^{\prime \prime}(t)=h \int_{D} W^{h-1}\left(\frac{\partial W}{\partial t}\right)^{2} d x+\int_{D} \frac{\partial^{2} W}{\partial t^{2}} d x
$$

To take equation of (6) into (13) and apply subsection integral, we obtain

$$
\Phi^{\prime \prime}(t)=h \int_{D} W^{h-1}\left(\frac{\partial W}{\partial t}\right)^{2} d x+\int_{\partial D} W^{h} \sum_{i, j=1}^{n}\left(a_{i j} \frac{\partial^{2} W}{\partial x_{j} \partial t}\right) n_{i} d s
$$




$$
\begin{gathered}
-h \int_{D} W^{h-1} \sum_{i, j=1}^{n} a_{i j} \frac{\partial^{2} W}{\partial x_{j} \partial t} \frac{\partial W}{\partial x_{i}} d x+\int_{D} b W \frac{\partial W}{\partial t} \ln W d x \\
+\int_{D} W^{h} \frac{\partial W}{\partial t} f d x+\int_{D} b W^{h} \frac{\partial W}{\partial t} d x+\int_{D} W^{h+1} f_{t}^{\prime} d x \\
+\int_{D} W^{h} \frac{\partial W}{\partial t} f_{\ln W}^{\prime} d x+\int_{D} W^{h+1} f_{\nabla_{x}}^{\prime} \ln W \\
\hline t
\end{gathered}
$$

Using 2 to multiply (14) and then subtract (12), with attention to the boundary condition in the (6) then obtain

$$
\begin{gathered}
\Phi^{\prime \prime}(t)=2 h \int_{D} W^{h-1}\left(\frac{\partial W}{\partial t}\right)^{2} d x+\int_{\partial D} \frac{W^{h}}{\beta}\left[g_{\ln W}^{\prime}+(h-1) \ln W-1\right] \frac{\partial W}{\partial t} d s \\
+(1-h) \int_{\partial D} \frac{W^{h}}{\beta} g \frac{\partial W}{\partial t} d s+\int_{\partial D} \frac{W^{h+1}}{\beta} g_{t}^{\prime} d s+\int_{\partial D} \frac{W^{h+1}}{\beta} g_{\nabla_{x} \ln W}^{\prime} \cdot \frac{\partial\left(\nabla_{x} \ln W\right)}{\partial t} d s+\int_{D} b W^{h} \frac{\partial W}{\partial t} d x \\
+h(h-1) \int_{D} W^{h-2} \frac{\partial W}{\partial t} \sum_{i, j=1}^{n} a_{i j} \frac{\partial W}{\partial x_{j}} \frac{\partial W}{\partial x_{i}} d x+\int_{D} W^{h+1} f_{t}^{\prime} d x \\
+\int_{D} W^{h+1} f_{\nabla_{l} n W}^{\prime} \cdot \frac{\partial\left(\nabla_{x} \ln W\right)}{\partial t} d x+\int_{D} W^{h} \frac{\partial W}{\partial t}\left[f_{\ln W}^{\prime}-(h-1) f\right] d x+(h-1) \int_{D} W^{h} \frac{\partial W}{\partial t} b \ln W d x
\end{gathered}
$$

According to supposed condition A1)—A4) and lemma1, lemma2 concerning the conclusion of non-negative $W$, $\frac{\partial W}{\partial t}$, we know

$$
\Phi^{\prime \prime}(t) \geq 2 h \int_{D} W^{h-1}\left(\frac{\partial W}{\partial t}\right)^{2} d x
$$

that is

$$
\Phi^{\prime \prime}(t) \cdot \Phi\left(t \geq \frac{2 h}{h+1} \int_{D} W^{h-1}\left(\frac{\partial W}{\partial t}\right)^{2} d x \cdot \int_{D} W^{h+1} d x \geq \frac{2 h}{h+1}\left(\int_{D} W^{h} \frac{\partial W}{\partial t} d x\right)^{2}=\frac{2 h}{h+1}\left[\Phi^{\prime}(t)\right]^{2}\right.
$$

cause

$$
J(t)=[\Phi(t)]^{-\frac{h-1}{h+1}}
$$

then through (9), (10) to know

$$
J(0)=[\Phi(0)]^{-\frac{h-1}{h+1}}>0, J^{\prime}(0)=-\frac{h-1}{h+1}[\Phi(0)]^{-\frac{2 h}{h+1}} \Phi^{\prime}(0)<0 .
$$

On the other side, through in equation (15) to know $J^{\prime \prime}(t) \leq 0$, According to Lemma 3, exists $T_{0}\left(0<T_{0}<-\frac{J(0)}{J^{\prime}(0)}\right)$, cause $J\left(T_{0}\right)=0$, we obtain

$$
\lim _{t \rightarrow T_{0}^{-}} J(t)=\lim _{t \rightarrow T_{0}^{-}}[\Phi(t)]^{-\frac{h-1}{h+1}}=0, \text { or } \lim _{t \rightarrow T_{0}^{-}} \Phi(t)=+\infty
$$

then we obtain

$$
\lim _{t \rightarrow T_{0}^{-}} \sup \{W(x, t), x \in \bar{D}\}=+\infty
$$

so

$$
\lim _{t \rightarrow T_{0}^{-}} \sup \{u(x, t), x \in \bar{D}\}=+\infty
$$

theorem has been proved.

\section{Applied example}

In the J. Bear's specialized work (Bcar., 1972), he proposed that the law of fluids in porous medium can be concluded to a quasi linear parabolic equation $u_{t}=\Delta\left(u^{m}\right)(m>1)$ We can put the $u<0$ situation aside because it does not exist in real life. Unfolding the equation and considering the initial boundary value problem:

$$
\left\{\begin{array}{lc}
\frac{\partial u}{\partial t}=m u^{m-1} \Delta u+m(m-1) u^{m-2}\left(\nabla_{x} u\right)^{2}, & D \times(0, T) \\
\frac{\partial u}{\partial v}+u=g(x, t) & \partial D \times(0, T) \\
u(x, 0)=u_{0}(x) & \bar{D}
\end{array}\right.
$$

Let $f\left(u, \nabla_{x} u\right)=m(m-1) u^{m-2}\left(\nabla_{x} u\right)^{2}$, then $f>0$.

The discussion below is to seek the conditions to the blowing up appearance of the solution of (17). 
By the supposed condition A2) it requests,

$$
f_{\nabla_{x} u}^{\prime} \cdot \frac{\partial\left(\nabla_{x} u\right)}{\partial t}=2 m(m-1) u^{m-2}\left(\nabla_{x} u\right) \cdot \frac{\partial\left(\nabla_{x} u\right)}{\partial t} \geq 0
$$

and when $h>1$,

$$
f_{u}^{\prime}-(h-1) f=m(m-1) u^{m-3}\left(\nabla_{x} u\right)^{2}[(m-2)-(h-1) u] \geq 0,
$$

and that get

$$
\left(\nabla_{x} u\right) \cdot \frac{\partial\left(\nabla_{x} u\right)}{\partial t} \geq 0
$$

and

$$
u \leq \frac{m-1}{h-1}
$$

By the condition A3), it requests, $g \leq 0, g_{t}^{\prime} \geq 0$ and $g_{u}^{\prime}+(h-1) u-1=(h-1) u-1 \geq 0$ then we get $u \geq \frac{1}{h-1}$. Considering it with (18) we get

$$
\frac{1}{h-1} \leq u \leq \frac{m-2}{h-1} \quad(m>3)
$$

Finality with the premise of A4), we get

$$
m\left(e^{u_{0}}\right)^{m-1} \triangle\left(e^{u_{0}}\right)+m(m-1)\left(e^{u_{0}}\right)^{m-2}\left(\nabla_{x} e^{u_{0}}\right)^{2}=m\left(e^{u_{0}}\right)^{m}\left[m\left(\nabla_{x} u_{0}\right)^{2}+\Delta u_{0}\right] \geq 0
$$

obviously $\Delta u_{0} \geq 0$ is the only prerequisite. According the discussion above we get the conclusion:

If the initial boundary of problem(4.1) given functions satisfies $g \leq 0, g_{t}^{\prime} \geq 0$ and $\Delta u_{0} \geq 0 . u(x, t)$ is the solution of (17) when it satisfies the inequalities of (18), (20). Then there must be definite time $T_{0}\left(0<T_{0}<+\infty\right)$, it makes blow up at $T_{0}$. The blowing up appearance help us to think about that the model of fluids low is reasonable and solvable.

\section{References}

Bcar. (1972). Dynamics of fluid in porous media, Amer. Elsevier, New York.

Chipot M., Werssler F.B. (1989). Some blow up results for a nonlinear parabolic equations with a gradient term. SIAM J. Math, Anal, 20(40), 886-907.

Deng, Jucheng. (1987). Some action-blowing up behavior of solution for pervasion equation. Applicable Mathematic Transaction, 10 (4) 450-456 (in Chinese).

Gomez J.L., Wolanski N. (1991). Blow-up results and localization of blow-up points for the heat equation with a nonlinear boundary condition. J. Diff, Eqs., 92 (2) 384-401.

Guan, Zhicheng. (1984). Some blowing up of solution for nonlinear parabolic equation. Chinese Annals of Mathematics, 5A(2), 177-180 (in Chinese).

Li, Junfeng, Lui, Weian, Lu, Gang. (2002). Global existence and blow up of sign-changing solutions in semi linear parabolic equations. Mathematic Physics Transaction, 22A (2), 150-156 (in Chinese).

Migoguchi N., Yanagida E. (1997). Critical exponent for the blow up of solutions with sign changes in a semi linear parabolic equation. Math. Ann., 307: 663-675.

Pao C.V. (1980). On the blowing up behavior of solutions for a parabolic boundary value problem. Applicable Analysis, Vol. 10, 5-13.

Zha, Zhongwei. (1992). The blowing up of solution for initial boundary value problem of semi-linear parabolic equations. Mathematica Applicata, 5(1), 82-87 (in Chinese).

Zhang, Hailiang, Jia, Xinchuen. (2002). Blowing-up of solution for quasi linear parabolic equations with a nonlinear boundary condition. Math. J., 22 (2), 195-198 (in Chinese).

Zheng, Zongmu, Chen, Yunmei. (1987). Blow up of nonlinear Developmental equation original boundary value problem. Journal of Fudan University (Natural Science Edition), 1, 19-27 (in Chinese). 